\title{
Detection of Entamoeba Species: A Comparative Analysis of Nested- Multiplex PCR and Recombinase Polymerase Amplification
}

\author{
Selvaratthinam Ajay Philips, Kumar Manochitra, Shashiraja Padukone and \\ Subhash Chandra Parija*
}

Department of Microbiology, Jawaharlal Institute of Postgraduate Medical Education and Research (JIPMER), Puducherry, India

*Corresponding author

\section{A B S T R A C T}

Keywords

Entamoeba histolytica, Species, Nested multiplex PCR

Article Info

Accepted:

16 February 2018

Available Online:

10 March 2018

Amoebiasis, caused by Entamoeba histolytica, remains a serious public health issue worldwide. For decades, microscopy remains preferred method for detection of the pathogenic species. The recent advent of molecular biology has helped in developing appropriate diagnostic technologies, of which isothermal amplification is considered to be robust and cost-effective compared to PCR that requires expensive instruments and highly skilled personnel. The aim of the present study was to compare the diagnostic ability of nested-multiplex PCR and Recombinase polymerase amplification for differential detection of E. histolytica and E. dispar. The results of this study showed good agreement between the two tests. This suggests that RPA can be used as clinical and epidemiological tool for diagnosis of amoebiasis in resource-limited setups and in endemic regions.

\section{Introduction}

Entamoeba histolytica causes amoebiasis which is globally considered as a leading parasitic cause of human morbidity and mortality (Haque et al., 2003; Haque and Petri, 2006). Clinically, E. histolytica manifestation ranges from asymptomatic colonisation to amoebic dysentery and invasive extraintestinal amebiasis, which is presented most commonly in the form of liver abscesses. Reports suggest that approximately 50 million people suffer from invasive amoebiasis, resulting in 100,000 deaths per year (WHO, 1997). E. histolytica is distributed worldwide; however, high prevalence rates have been reported from various developing and under-developed countries (Fotedar, 2007).

Entamoeba histolytica, Entamoeba dispar, Entamoeba moshkovskii, Entamoeba coli, Entamoeba hartmanni, and Entamoeba polecki are the six species among the Genus Entamoeba that are found to reside in the human intestinal lumen. Recent studies have 
isolated E. dispar and E. moshkovskii from individuals with gastrointestinal symptoms. The casual link between these parasites that are considered non-pathogenic and the disease is still not evident (Clark and Diamond, 1991). Until obvious evidence is established, the detection and differentiation of the different Entamoeba sp. is essentially important. Appropriate methodologies for specific diagnosis of amoebiasis in endemic areas are highly recommended because of the morphologically indistinguishable characteristics of E. histolytica, E. dispar and E. moshkovskii.

For many years, diagnosis of $E$. histolytica was primarily based on microscopic examination of the protozoan morphology. However, its sensitivity reaches up to only $60 \%$ and there can be issues with misidentification of non-pathogenic Entamoeba species. Unless the trophozoites contain ingested RBCs, the ability to differentiate E. histolytica from other lookalike species becomes technically tricky (Gonzalez-Ruiz et al., 1994; Manochitra and Parija, 2017).

Recently molecular biology has revolutionized the field of pathogen identification and differentiation based on the difference in the genetic make-up. The sensitivity and the chances of specific identification have been improved by application of the molecular methods like polymerase chain reaction (PCR) assays. Nested multiplex PCR (NM-PCR) performed from faecal and liver abscess aspirate specimens, targeting the $18 \mathrm{~S}$ SSU rRNA has been evaluated for diagnosing amoebiasis (Khairnar and Parija, 2007). However, it is time-consuming and requires expensive equipment for thermal cycling thus limiting their use in resource-poor setups. Isothermal DNA amplification platforms, such as the Recombinase polymerase amplification (RPA) used in this study, alleviate the need for thermal cycling equipment and have the potential to broaden access to more sensitive diagnostics. RPA doesn't require thermocyclers to provide robust signal amplification. In RPA, the target sequence is scanned in the DNA template based on the interaction of the oligonucleotide primer with a nucleoprotein complex formed by a recombinase and its co-factor. The beginning of the strand invasion is marked by the recognition of a specific homologous sequence followed by extension of the opposing nucleotides by isothermal strand displacement amplification (Daher et al., 2016). Based on the benefits of RPA over PCR, it has gained importance in the specific diagnosis of various diseases. In the present study, we have compared the diagnostic ability of NM-PCR and RPA for accurate detection and differentiation of Entamoeba sp.

\section{Materials and Methods}

This study was primarily hospital-based and was carried out over a period of one year (2016-2017) in the Department of Microbiology, JIPMER, India. The stool samples $(n=180)$ from patients presenting with clinical symptoms of amoebic colitis like diarrhoea/dysentery, the presence of abdominal pain with or without the onset of fever and other gastrointestinal disturbances were randomly included in the study after obtaining clearance from JIPMER Institute Ethics Committee. The samples were stored at $-20^{\circ} \mathrm{C}$ without the addition of any preservatives or fixatives until used.

\section{DNA extraction and nested multiplex PCR}

DNA was extracted from the stored stool specimens according to the manufacturer's instructions with the QIAamp Stool DNA Mini Kit. The NM-PCR with the primer targeting the 18S SSU rRNA gene was carried out as previously described (Khairnar and 
Parija, 2007) in Sure Cycler 8800 (Agilent Technologies). The amplified product was visualised on $1.8 \%$ agarose in a gel documentation unit (GelDoc XR, BioRad).

\section{Recombinase polymerase amplification}

The RPA assays were performed using the TWISTAMP $^{\circledR}$ basic kit (TwistDx, Cambridge, United Kingdom) following the manufacturer's instructions. Amplifications were performed based on the previously described protocol with some minor modifications (Nair et al., 2015). A set of forward primers specific for $E$. histolytica (EHF 5'GTACAAAATGGCCAATTCAT TCAAT3') and E. dispar (EDF 5' AAGTATAAAGACCAAGTAGGATGAAA3 ') and common reverse primer (EHEDR 5'ACTACCAACTGATTGATAGATCAG3') targeting the 18S SSU rRNA gene were used. Each primer set could amplify $132 \mathrm{bp}$ product for E. histolytica (EHF/EHEDR) and $234 \mathrm{bp}$ product for $E$. dispar (EDF/EHEDR). The amplified product was purified, and $5 \mu \mathrm{L}$ of each RPA reaction was analyzed by electrophoresis in 1\% agarose gel followed by ethidium bromide staining for visualization under UV trans-illuminator.

\section{Results and Discussion}

A total of 180 stool samples were subjected to NM-PCR and RPA. The species-specific NMPCR product size for E. histolytica and $E$. dispar was 439 and 174 bp respectively (Figure 1). Of the total 180 samples, 13 were positive by NM-PCR, of which 11 were found to be E. histolytica, and only 2 were $E$. dispar (Table 1).

After amplification by RPA which yielded products of 132 bp size for E. histolytica and 234 bp size for $E$. dispar were visualized on $1 \%$ agarose gel (Figure 2). Amplified products could be visualised in a total of 11 samples, of which 9 were positive for E. histolytica and 2 for E. dispar (Table 1).

Based on the results, the sensitivity and specificity of RPA were statistically calculated, which was found to be $84.62 \%$ and $100 \%$ respectively; the positive predictive values and negative predictive values for RPA were $100 \%$ and $98.83 \%$ respectively when compared with NM- PCR (Table 2). The previous report shows that the sensitivity and specificity of NM-PCR to be $94 \%$ and $100 \%$ (Khairnar and Parija, 2007). On the other hand the Kappa agreements between the two tests were found to be very good.

Recently in the developed countries, PCR has been the method of choice for clinical and epidemiological studies and has been strongly recommended to be used as a diagnostic tool by the WHO (Haque, 2006; Khairnar and Parija, 2007; Tanyuksel and Petri, 2003; Calderaro et al., 2006; Hamzah et al., 2006; Zaki et al., 2002).

A variety of clinical specimens (faeces, tissues, saliva, liver abscess aspirate) have been tested for identification of E. histolytica (Khairnar and Parija, 2008) various diagnostic methods are being developed for rapid, specific and differential detection of the Entamoeba sp., (Parija et al., 2014; Korpe et al., 2012) which may aid in formulating appropriate treatment strategies. Isothermal amplification methods are robust and are currently in the developmental stage for the diagnosis of various pathogens. RPA has been tested for parasites such as Plasmodium, Cryptosporidium, Schistosoma haematobium and few other bacteria and viruses (JausetRubio et al., 2016). There have been very few reports about utilization of RPA for diagnosis of amoebiasis and they are mainly used in research laboratories (Nair et al., 2015; Crannell et al., 2016). 
Table.1 Comparison of NM PCR and RPA

\begin{tabular}{|l|l|l|l|l|}
$\begin{array}{l}\text { Samples } \\
\text { N=180 }\end{array}$ & $\begin{array}{l}\text { Positive by NM } \\
\text { PCR }\end{array}$ & $\begin{array}{l}\text { Positive } \\
\text { RPA }\end{array}$ \\
\hline & EH & ED & EH & ED \\
\hline & $\mathbf{1 1}$ & $\mathbf{2}$ & $\mathbf{9}$ & $\mathbf{2}$ \\
\hline Total & & $\mathbf{1 3}$ & $\mathbf{1 1}$ & \\
\hline
\end{tabular}

Table.2 Two-way contingency table for comparing RPA against NM PCR

\begin{tabular}{|c|c|c|c|}
\hline & NM PCR Positive & NM PCR Negative & Total \\
\hline RPA Positive & 11 & $\mathbf{0}$ & 11 \\
\hline RPA Negative & 2 & 167 & 169 \\
\hline Total & 13 & 167 & 180 \\
\hline
\end{tabular}

Sensitivity and Specificity was calculated by using Medcalc. Kappa agreement was calculated by graph pad.

Fig.1 PCR products of NM-PCR in $1.8 \%$ agarose gel

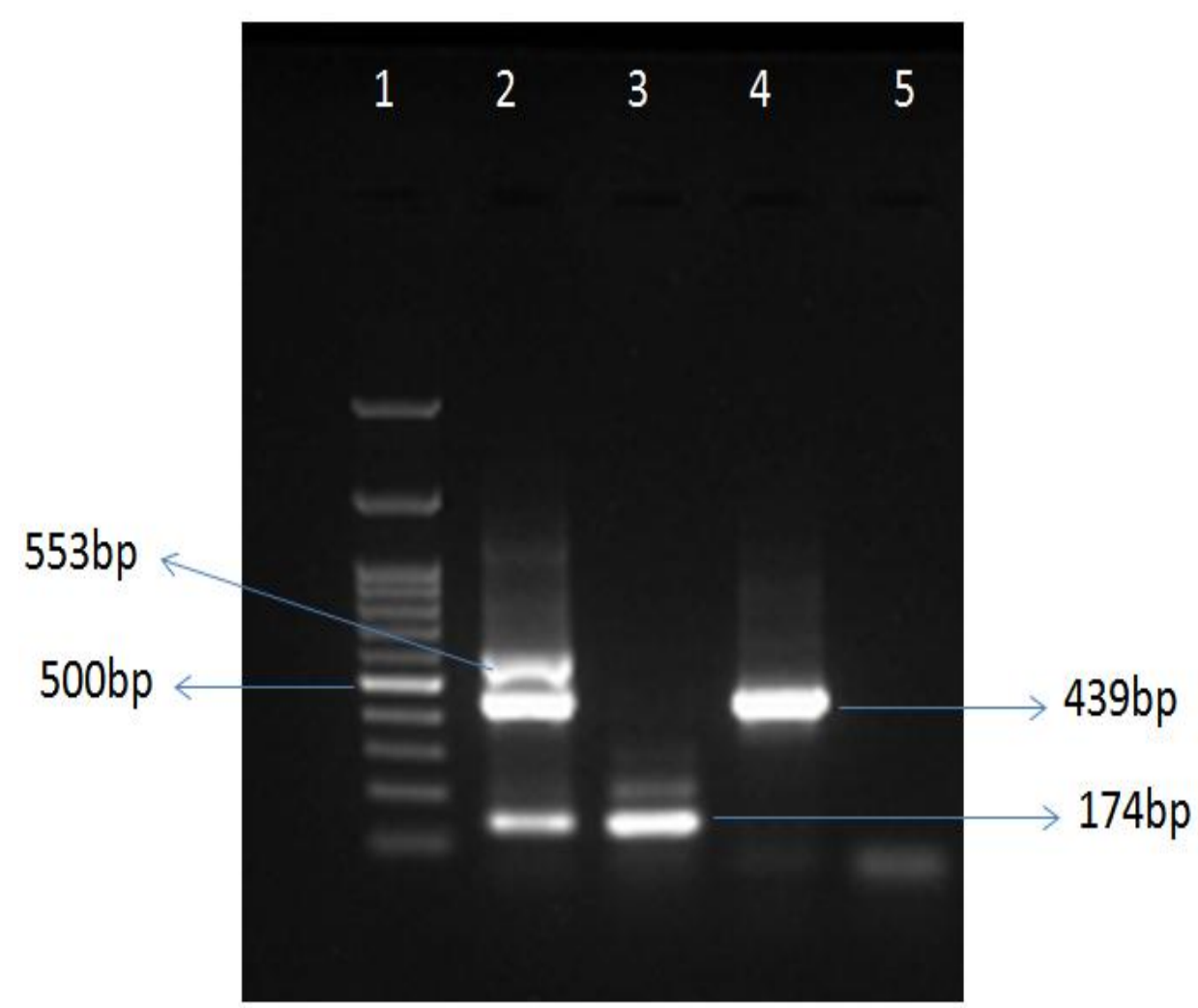

Lane 1- 100bp Marker, Lane 2- positive control (EH-439bp, ED-174bp, EM-553bp) Lane-3\&4 sample, Lane 5negative control 
Fig.2 RPA products in 1\% agarose gel

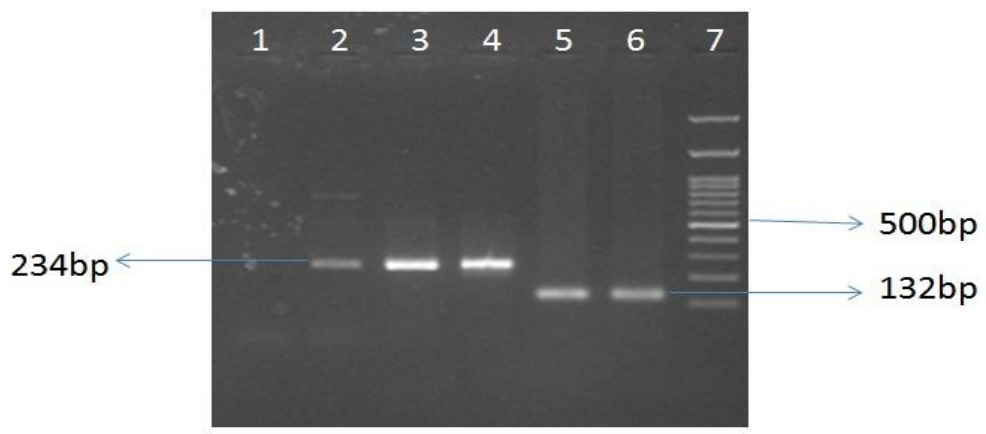

Lane 1- negative control, Lane $2 \& 3$ samples, Lane-4 -Positive control(ED), Lane-5 - sample, Lane-6-Positive control (EH), Lane-7-100bp Marker

The study by Nair et al., (2015) has tested the ability of RPA for utilization in the detection of the Entamoeba sp., and they found that RPA showed $86 \%$ and $98 \%$ correlation with the positive and negative samples which were screened by PCR and ELISA. A study by Crannell et al., (2016) has attempted to develop a multiplex assay using RPA for intestinal parasites like Giardia, Entamoeba and Cryptosporidium. In our study, we have directly tested clinical specimens and have successfully differentiated $E$. dispar from $E$. histolytica.

As RPA for E. moshkovskii has not been studied so far, we have used it only for detection of $E$. histolytica and E. dispar. Considering this, for comparative purpose the NM-PCR parameters were slightly modified, wherein the primers for E. moshkovskii were not included.

RPA eliminates the need for thermocyclers, thereby reducing the high cost spent on the equipment. This enhances its utility in developing and under-developed countries where the disease burden is high. RPA when combined with lateral flow (LF) techniques, makes it an efficient point-of-care test.

Future studies combining RPA with LF and testing it on larger samples may help in evaluating its ability as an epidemiological tool in endemic areas.
RPA is a simple, rapid and efficient diagnostic method that can be used in both clinical and epidemiological studies. The nominal cost and efficiency are the important factors that have to be considered in widely implementing this method. This study strongly suggests that RPA could be a valuable tool in resource poor settings if developed in-house so as to bring down the cost.

This study represents the first effort to evaluate RPA in comparison with NM PCR in an parasite endemic region like India.

\section{Acknowledgments}

This work was supported by JIPMER intramural Fund.

\section{References}

Calderaro A, Gorrini C, Bommezzadri S, Piccolo G, Dettori G, Chezzi C. 2006. Entamoeba histolytica and Entamoeba dispar: comparison of two PCR assays for diagnosis in a non-endemic setting. Trans. R. Soc. Trop. Med. Hyg. 100:450457.

Clark CG, Diamond LS. 1991. Ribosomal RNA genes of 'pathogenic' and 'nonpathogenic' Entamoeba histolytica are distinct. Mol. Biochem. Parasitol. 
9:297-302.World Health Organization. 1997. Amebiasis. Wkly. Epidemiol. Rec. 72:97-100.

Crannell Z, Castellanos-Gonzalez A, Nair G, Mejia R, White AC, Richards-Kortum R. Multiplexed Recombinase Polymerase Amplification Assay To Detect Intestinal Protozoa. Anal Chem. 2016348:1565-73.

Daher RK, Stewart G, Boissinot M, Bergeron MG. 2016. Recombinase Polymerase Amplification for Diagnostic Applications. Clin Chem., 62: 947-58.

Fotedar R, Stark D, Beebe N, Marriott D, Ellis J, Harkness J. 2007. A review of laboratory diagnostic techniques for Entamoeba species. Clin. Microbiol. Rev.; 20:511-532.

González-Ruiz A, Haque R, Aguirre A, Castañón G, Hall A, Guhl F, RuizPalacios G, Miles MA, Warhurst DC. 1994. Value of microscopy in the diagnosis of dysentery associated with invasive Entamoeba histolytica. J Clin Pathol. 47:236-9.

Hamzah Z, Petmitr S, Mungthin M, Leelayoova S, Chavalitshewinkoon-Petmitr P, 2006. Differential detection of Entamoeba histolytica, Entamoeba dispar, and Entamoeba moshkovskii by a single-round PCR assay. J. Clin. Microbiol. 44:31963200.

Haque R, and Petri, WA. Jr. 2006. Diagnosis of amebiasis in Bangladesh. Arch. Med. Res.; 37:273-276.

Haque R, Huston CD, Hughes M, Houpt E, Petri WA Jr., 2003. Amebiasis. N Engl J Med.

Jauset-Rubio M, Svobodová M, Mairal T, McNeil C, Keegan N, Saeed A, Abbas MN, El-Shahawi MS, Bashammakh AS, Alyoubi AO, O Sullivan CK. 2016. Ultrasensitive, rapid and inexpensive detection of DNA using paper based lateral flow assay. Sci Rep. 6:37732

Khairnar K, Parija SC. 2002. Detection of Entamoeba histolytica DNA in the saliva of amoebic liver abscess patients who received prior treatment with metronidazole. J Health Popul Nutr.; 26: 418-425.

Khairnar K, Parija SC. 2007. A novel nested multiplex PCR assay for differential detection of Entamoeba histolytica, E. moshkovskii and E. dispar DNA in stool samples. BMC Microbiol. 7:47.

Korpe PS, Stott BR, Nazib F, Kabir M, Haque R, Herbein JF, Petri WA Jr. 2012. Evaluation of a rapid point-of-care fecal antigen detection test for Entamoeba histolytica. Am J Trop Med Hyg. 86:9801

Manochitra K, Parija SC. 2017 Comparison of microscopy and enzyme-linked immunosorbent assay for detection of the pathogenic Entamoeba sp., from fecal specimens. European Journal of Biotechnology and Bioscience. 5:17-19

Nair G, Rebolledo M, White AC Jr, Crannell Z, Richards-Kortum RR, Pinilla AE. 2015. Detection of Entamoeba histolytica by Recombinase Polymerase Amplification. Am J Trop Med Hyg. 93:591-5.

Parija SC, Mandal J, Ponnambath DK. 2014. Laboratory methods of identification of Entamoeba histolytica and its differentiation from look-alike Entamoeba spp. Trop Parasitol.4:90-5.

Tanyuksel M, Petri Jr WA. 2003. Laboratory diagnosis of amebiasis. Clin. Microbiol. Rev.16:713-729.

Zaki M, Meelu P, Sun W, Clark CG. 2002. Simultaneous differentiation and typing of Entamoeba histolytica and Entamoeba dispar. J. Clin. Microbiol. 40:1271-1276.

\section{How to cite this article:}

Selvaratthinam Ajay Philips, Kumar Manochitra, Shashiraja Padukone and Subhash Chandra Parija. 2018. Detection of Entamoeba Species: A Comparative Analysis of Nested-Multiplex PCR and Recombinase Polymerase Amplification. Int.J.Curr.Microbiol.App.Sci. 7(03): 1803-1808. doi: https://doi.org/10.20546/ijcmas.2018.703.212 